\title{
The pressure accuracy of fractional-step methods for the Navier-Stokes equations on staggered grids
}

\author{
S. Armfield ${ }^{*} \quad$ R. Street ${ }^{\dagger}$
}

(Received 1 June 2001, revised September 2002)

\begin{abstract}
Previous investigations of the time accuracy of the pressure obtained with fractional step methods have shown it to be first order in time, irrespective of the order of accuracy of the method. In this paper a detailed analysis and investigation of the pressure accuracy for P1 and P2 type fractional step methods on staggered finite volume grids is carried out. It is shown that the standard P1 scheme produces a pressure that is first order accurate in time, as noted by previous investigators. However the standard P2 scheme is shown to give a second order accurate in time pressure. It is shown
\end{abstract}

*School of Aerospace, Mechanical \& Mechatronic Engineering, Sydney University, Sydney, Australia. mailto: armfield@mech.eng.usyd.edu.au

$\dagger$ Environmental Fluid Mechanics Laboratory, Stanford University, Stanford, California 94305-4020, USA

${ }^{0}$ See http://anziamj.austms.org.au/V44/CTAC2001/Armf for this article,

(C) Austral. Mathematical Soc. 2003. Published 1 April 2003. ISSN 1446-8735 
that the first order behaviour observed by previous investigators for P2 schemes was an artifact of the means of testing the accuracy. Finally it is shown that second order pressure is obtained with the P1 method by using a corrected full pressure.

\section{Contents}

1 Introduction

2 Numerical Method

2.1 Iterative method. . . . . . . . . . . . C 24

2.2 P2 Non-iterative method . . . . . . . . . . C C27

2.3 P1 Non-Iterative method . . . . . . . . . . C28

2.4 Boundary Conditions and Discretisation .... . C 30

3 Results

C32

4 Conclusions

C36

References

C38

\section{Introduction}

Fractional step methods integrate the Navier-Stokes equations in time at each time-step by first solving the momentum equations using an approximate pressure field to yield an intermediate velocity field that will not, in general, satisfy continuity. A Poisson equation is then solved with the divergence of the intermediate velocity as a source term to provide a pressure or pressure correction, which is 
then used to correct the intermediate velocity field, providing a divergence free velocity. The pressure is updated and integration then proceeds to the next time step. Details of the range of fractional step methods developed for the Navier-Stokes equations are given in $[1,2,3,4,5,6,7,8,9,10,11,12,13]$. The P1 method sets the pressure field to zero in the momentum equation and the Poisson equation is then solved for the new pressure, while the P2 method sets the pressure in the momentum equation to that obtained at the previous time-step, and the Poisson equation is then solved for a pressure correction. Both the P1 method, with appropriate intermediate velocity boundary conditions, and the P2 method provide second order in time accuracy for the velocity field, provided the momentum equation is integrated using a second order accurate scheme. However a number of investigations have shown the pressure to be only first order accurate in time, irrespective of the accuracy of the velocity $[6,7,13]$. Typically the time accuracy is examined by obtaining the difference between a series of test solutions with varying time-steps and a benchmark solution obtained with a very small time-step, integrated to the same time.

In this paper the time-accuracy of the pressure is examined in detail. It is shown that the first-order accuracy obtained with the P1 scheme is an inherent feature of the scheme, however the apparent first order accuracy of the pressure obtained with the P2 scheme is an artifact of the means by which the accuracy has been tested, and the P2 scheme does provide second-order in time accuracy in both the velocity and pressure fields. 


\section{Numerical Method}

The Navier-Stokes equations in unsteady incompressible non-dimensional form are,

$$
\begin{aligned}
u_{t}+(u \cdot \nabla) u & =-\nabla P+\frac{1}{\mathrm{Re}} \nabla^{2} u, \\
\nabla \cdot u & =0,
\end{aligned}
$$

where $u$ is the velocity, $P$ the pressure and Re the Reynolds number. The continuous equations are discretised using Adams-Bashforth for the advective terms and Crank-Nicolson for the diffusive terms, giving the system

$$
\begin{aligned}
& \frac{v^{n+1}-v^{n}}{\Delta t}+\left[\frac{3}{2} H\left(v^{n}\right)-\frac{1}{2} H\left(v^{n-1}\right)\right] \\
& =-G p+\frac{1}{2 \operatorname{Re}} L\left(v^{n+1}+v^{n}\right), \\
& D v^{n+1}=0,
\end{aligned}
$$

where $(v, p)$ are the discrete velocity and pressure respectively, $H$ is the discrete advection operator, $G$ the discrete gradient, $L$ the discrete Laplace operator and $D$ the discrete divergence. The time derivative has been discretised using a centred approximation, and thus is second order accurate at the time location $n+1 / 2$. The advection terms have been discretised using an explicit AdamsBashforth approximation as,

$$
H\left(v^{n+1 / 2}\right) \sim \frac{3}{2} H\left(v^{n}\right)-\frac{1}{2} H\left(v^{n-1}\right)+O\left(\Delta t^{2}\right),
$$

also giving second order time accuracy at the time location $n+$ $1 / 2$. The symbol $\sim$ is used here and below to indicate that higher order terms in $\Delta t$ have been dropped. The viscous terms have been discretised using Crank-Nicolson, with again second order in time 
accuracy at time location $n+1 / 2$. Equation (3) is therefore a second order in time representation of equation (1) at the $n+1 / 2$ time location.

Fractional-step methods integrate equations (3) and (4) in a segregated manner, that is the momentum equations are first solved for the velocity, and some form of Poisson equation is then solved for the pressure. The Poisson equation is constructed from the momentum equation and the continuity equation and, as well as providing the pressure, acts to enforce continuity.

\subsection{Iterative method.}

In this method equation (3) is solved, using the best current value for $p$, to obtain $v^{*}$, an approximation to $v^{n+1}$, that is

$$
\frac{v^{*}-v^{n}}{\Delta t}+\left[\frac{3}{2} H\left(v^{n}\right)-\frac{1}{2} H\left(v^{n-1}\right)\right]=-G p+\frac{1}{2 \operatorname{Re}} L\left(v^{*}+v^{n}\right) .
$$

This approximate velocity will not initially satisfy continuity. A correction is then applied of the form,

$$
v^{n+1}=v^{*}-\Delta t G \pi,
$$

where $\pi$ is a pressure correction, such that the resulting $v^{n+1}$ does satisfy continuity. An equation for $\pi$ is constructed by substituting equation (6) into the continuity equation (4), to give,

$$
L \pi=D v^{*} / \Delta t .
$$

Once $\pi$ is obtained, the velocity is corrected and the pressure is updated using the pressure correction as,

$$
p_{\text {new }}=p+\pi,
$$


where $p_{\text {new }}$ is the updated pressure. Equation (5) is then solved again using the updated pressure to obtain a new estimate of the velocity at the $n+1$ time level, and that velocity again corrected to enforce continuity and provide a pressure correction. This process is repeated until the integral over the domain of the absolute divergence after the solution of equation (5) is less than a predefined value. The solution is then said to be converged and the integration continues to the next time step. For the first iteration at each timestep $p$ is set equal to the stored pressure obtained from the previous time-step.

In practice the scheme is most efficient if it is not required that the pressure correction step results in a velocity field that satisfies the divergence free condition at each iteration, rather it is only required that the divergence error be reduced, and over a number of momentum/pressure correction iterations the velocity will approach and satisfy the divergence free condition.

Pressure Accuracy The pressure obtained with the iterative method satisfies the discrete Poisson equation obtained by taking the discrete divergence of equation (5), that is,

$$
L p=-D\left[\frac{3}{2} H\left(v^{n}\right)-\frac{1}{2} H\left(v^{n-1}\right)\right],
$$

where the time derivative and viscous terms have been dropped using $D(v)=0$. The right hand side of this equation is a second order extrapolation of the divergence of advection from time levels $n$ and $n-1$ to $n+1 / 2$. Given that the velocity $v$ is second order accurate in time at time levels $n$ and $n-1$, the right hand side of this equation may be represented as,

$$
D\left[\frac{3}{2} H\left(v^{n}\right)-\frac{1}{2} H\left(v^{n-1}\right)\right] \sim D\left[H\left(v^{n+1 / 2}\right)\right]+O\left(\Delta t^{2}\right)
$$


and hence the right hand side of the pressure equation is second order accurate in time at time location $n+1 / 2$, indicating that the pressure is second order accurate in time at time level $n+1 / 2$, and should be denoted $p^{n+1 / 2}$.

Previous investigations have examined the pressure accuracy by obtaining a small time step solution which is used as an approximation of the exact solution. The pressure obtained at larger time steps is then compared to the exact solution to determine the order of accuracy of the scheme. The problem with this approach is as follows. Suppose the small time step solution is obtained with $\Delta t_{f}=0.01$ integrated for $N_{f}=100$ timesteps to $t^{N_{f}}=1$, and the test solution is obtained with $\Delta t_{t}=0.1$ integrated for $N_{t}=10$ time steps to $t^{N_{t}}=1$. The final pressure solution is second order accurate in time at $N_{t} \Delta t_{t}-\Delta t_{t} / 2$ on the test time-step, and at $N_{f} \Delta t_{f}-\Delta t_{f} / 2$ on the fine time-step, however there is always a $\Delta t_{t} / 2-\Delta t_{f} / 2$ difference between these two time locations, which will introduce an order $\Delta t_{t}$ error when the pressures are compared.

To verify the second-order in time pressure accuracy the number of time-steps used for the fine and test solutions should be chosen so that $\left(N_{t} \Delta t_{t}-\Delta t_{t} / 2\right)=\left(N_{f} \Delta t_{f}-\Delta t_{f} / 2\right)$. Alternatively the $n+1 / 2$ and $n-1 / 2$ pressure solutions can be extrapolated using a second order scheme to give an $n+1$ time level pressure, which can then be tested for accuracy in the standard way. In this paper the latter of these approaches is used, with the pressure extrapolation,

$$
p^{n+1}=\frac{3}{2} p^{n+1 / 2}-\frac{1}{2} p^{n-1 / 2},
$$

where the $p^{n+1 / 2}$ and $p^{n-1 / 2}$ pressures are those obtained directly from the solution procedure. This is a second order extrapolation of pressure from time levels $n+1 / 2$ and $n-1 / 2$ to $n+1$ and, given that the pressure is second order accurate in time at $n+1 / 2$ 
and $n-1 / 2$, will give a second order accurate approximation to the pressure at $n+1$.

\section{$2.2 \quad$ P2 Non-iterative method}

The P2 non-iterative method is identical to the iterative method, but with only a single iteration carried out at each time step. The discrete momentum equation is solved to obtain $v^{*}$, that is

$$
\frac{v^{*}-v^{n}}{\Delta t}+\left[\frac{3}{2} H\left(v^{n}\right)-\frac{1}{2} H\left(v^{n-1}\right)\right]=-G p^{n-1 / 2}+\frac{1}{2 \operatorname{Re}} L\left(v^{*}+v^{n}\right),
$$

where the $n-1 / 2$ time-level pressure is that obtained at the previous time-step. The $v^{*}$ field is then corrected to satisfy continuity and the pressure corrected in exactly the same way as in the iterative method. As the pressure correction equation is only solved once each time step with the non-iterative approach, it is necessary to obtain an accurate solution of the Poisson equation.

Pressure accuracy To derive the Poisson equation satisfied by the pressure obtained with the non-iterative scheme it is necessary to substitute the intermediate velocity in the form given in equation (6) into the momentum equation (10), in terms of the pressure correction $\pi$ and the $n+1$ level velocity, giving,

$$
\begin{aligned}
& \frac{v^{n+1}-v^{n}}{\Delta t}+\left[\frac{3}{2} H\left(v^{n}\right)-\frac{1}{2} H\left(v^{n-1}\right)\right] \\
& =-G\left(p^{n-1 / 2}+\pi\right)+\frac{\Delta t}{2 \operatorname{Re}} L G \pi+\frac{1}{2 \operatorname{Re}} L\left(v^{n+1}+v^{n}\right) .
\end{aligned}
$$


Setting $p^{n+1 / 2}=p^{n-1 / 2}+\pi$, and taking the divergence of this equation, gives,

$$
L p^{n+1 / 2}=-D\left[\frac{3}{2} H\left(v^{n}\right)-\frac{1}{2} H\left(v^{n-1}\right)+\frac{\Delta t}{2 \operatorname{Re}} L G \pi\right] .
$$

The first two components of the source term on the right hand side of this equation are the same as those obtained with the iterative method, above, and again represent a second order accurate in time approximation for the divergence of the advection at $n+1 / 2$. The third term is an error associated with the pressure approximation of the non-iterative scheme, but as the pressure correction, $\pi$, is first order in time, this term is also second order in time. The pressure obtained using the standard non-iterative pressure correction scheme is therefore second order accurate in time at $n+1 / 2$, as for the iterative scheme. Once again to obtain a second order accurate $p^{n+1}$ it is necessary to use the extrapolation given above in equation (9). Additionally since the operators $G$ and $L$ commute in the interior of the domain a more accurate pressure may be obtained by setting

$$
p^{n+1 / 2}=p^{n-1 / 2}+\pi-\frac{\Delta t}{2 \operatorname{Re}} L \pi,
$$

which is also second order in time and is denoted the full pressure.

\section{$2.3 \quad$ P1 Non-Iterative method}

In this method equation (5) without the pressure gradient term is solved to obtain $v^{*}$, that is

$$
\frac{v^{*}-v^{n}}{\Delta t}+\left[\frac{3}{2} H\left(v^{n}\right)-\frac{1}{2} H\left(v^{n-1}\right)\right]=\frac{1}{2 \operatorname{Re}} L\left(v^{*}+v^{n}\right) .
$$


Again this approximate velocity field will not in general satisfy continuity and a correction is applied of the form,

$$
v^{n+1}=v^{*}-\Delta t G \phi,
$$

such that the resulting $v^{n+1}$ does satisfy continuity. An equation for $\phi$ is constructed by substituting equation (16) into equation (4), to give,

$$
L \phi=D v^{*} / \Delta t .
$$

Once $\phi$ is obtained, the velocity is corrected and the integration continues to the next time step. At each time step $\phi$ is initialised using the previous timestep value. This significantly reduces the run time, by up to a factor of two, when compared to the run time for $\phi$ initialised to zero at each time-step.

Pressure Accuracy Substituting the intermediate velocity in the form given in equation (16) into the momentum equation (15) for the P1 method gives,

$$
\begin{aligned}
& \frac{v^{n+1}-v^{n}}{\Delta t}+\left[\frac{3}{2} H\left(v^{n}\right)-\frac{1}{2} H\left(v^{n-1}\right)\right] \\
& =-G \phi+\frac{\Delta t}{2 \operatorname{Re}} L G \phi+\frac{1}{2 \operatorname{Re}} L\left(v^{n+1}+v^{n}\right) .
\end{aligned}
$$

Setting $p^{n+1 / 2}=\phi$, as is the normal practise with P1 methods, and taking the divergence of this equation, gives,

$$
L p^{n+1 / 2}=-D\left[\frac{3}{2} H\left(v^{n}\right)-\frac{1}{2} H\left(v^{n-1}\right)+\frac{\Delta t}{2 \operatorname{Re}} L G \phi\right] .
$$

The first two components of the right hand side of this equation are again a second order accurate approximation for the divergence of the advection. However the last term is now first order in time, and 
thus the pressure obtained with the standard approximation used in the P1 scheme will be first order. It is once again clear that a more accurate pressure can be obtained by setting

$$
p^{n+1 / 2}=\phi-\frac{\Delta t}{2 \operatorname{Re}} L \phi .
$$

which will again be denoted the full pressure. This pressure will satisfy the Poisson equation,

$$
L p^{n+1 / 2}=-D\left(\frac{3}{2} H\left(v^{n}\right)-\frac{1}{2} H\left(v^{n-1}\right)\right),
$$

indicating that for the P1 scheme the full pressure is second order in time. Thus it is expected that using the full pressure for the P1 scheme will provide a significantly more accurate pressure field than the standard pressure approximation.

\subsection{Boundary Conditions and Discretisation}

The above schemes are defined on the standard MAC staggered grid using finite volumes. Results are presented below for natural convection flow in a square cavity which requires fixed velocity boundary conditions. The normal component of velocity, which has a node on the boundary, is set to the required value at that boundary, while the tangential component, which does not have a node on the boundary, has the average of the values at the immediate interior and exterior nodes set to the required value. Because the normal component of velocity is known at the boundary no correction is required to the ${ }^{*}$ field there, and therefore the normal gradient of $\pi$ and $\phi$ is set to zero at the boundary. No explicit boundary conditions are required for the pressure. For the iterative solver the boundary conditions for the ${ }^{*}$ velocity field are set to be the same as the physical boundary conditions, given above. 
The P1 non-iterative solver requires a modified boundary condition for the ${ }^{*}$ tangential velocity field as follows,

$$
U_{1}^{*}=-U_{2}^{*}+\frac{2 \Delta t}{h} \Delta p_{1}^{n-1 / 2}
$$

where $U^{*}$ is the tangential velocity, subscript ${ }_{1}$ is the immediate domain exterior point, subscript 2 is the immediate domain interior point, $h$ is the grid size and $\Delta p$ is the $x$ difference of pressure. Physical velocity boundary conditions are applied to the intermediate normal boundary velocity. This boundary condition is similar to that developed by Kim and Moin [3] and is necessary to ensure second-order in time accuracy for the velocity for the P1 method [13]. The P2 method has second-order in time accuracy with the physical boundary conditions applied to the intermediate velocity field, however an improvement in accuracy is obtained using the Kim and Moin boundary condition given above, but with $\pi^{n-1 / 2}$ rather than $p^{n-1 / 2}$. Results will be obtained for both the standard boundary conditions and the Kim and Moin boundary conditions for the P2 method.

The equations are discretised using standard second-order central differences for the viscous terms, the pressure gradient and divergence terms. The QUICK third-order upwind scheme is used to obtained face values for use in the advective terms [14]. The momentum equations are inverted using an ADI scheme in which terms are shifted to the right hand side of the system to enable a series of tridiagonal matrices to be inverted in each direction. The terms shifted to the right hand side contain the latest available estimate for the unknown, allowing the domain to be repeatedly swept until an accurate solution is obtained. For all the methods tested four sweeps of the ADI solver were used, where a single sweep consists of solving the series of tridiagonal systems associated with each coordinate direction once. Four sweeps of the solver gave solutions with residuals of less than $10^{-8}$ for all cases. A preconditioned 
restarted GMRES method is used to solve the Poisson $\phi$ and pressure correction equations for all the methods. Other solvers, such as preconditioned conjugate gradient, incomplete LU, ADI and Jacobi have also been tested and found not to affect the overall accuracy or relative performance of the methods. Of the solvers tested GMRES was found to be the most efficient. The number of sweeps of the GMRES solver used varied with each of the methods tested and with the time-step and convergence criterion prescribed. For the non-iterative schemes for the smallest convergence criterion up to a hundred sweeps were required while for the largest convergence criterion as few as five were sufficient. For the iterative scheme the Poisson solver was limited to five sweeps. At each time step the solution was considered converged when the integral of the absolute divergence over the domain was less than a preset value. The convergence is applied to the iterative scheme after the solution of the momentum equations, with a minimum of two momentum/Poisson pressure correction iterations required.

\section{Results}

The results presented below for natural convection flow in a square cavity were obtained in double precision on a DEC 3000-700. The simulation of natural convection flow requires the solution of a temperature equation in addition to the momentum and Poisson pressure correction equations described above, and the governing equations then become,

$$
\begin{aligned}
T_{t}+(u \cdot \nabla) T & =\frac{1}{\mathrm{Ra}^{1 / 2}} \nabla^{2} T, \\
u_{t}+(u \cdot \nabla) u & =-\nabla P+\frac{\operatorname{Pr}}{\mathrm{Ra}^{1 / 2}} \nabla^{2} u+b \operatorname{Pr} T, \\
\nabla \cdot u & =0,
\end{aligned}
$$


where $T$ is the temperature and $b$ represents the gravity force in the buoyancy term, with $b=1$ for the vertical component of velocity and $b=0$ for the horizontal component of velocity. The control parameters for this flow are the Rayleigh number Ra and the Prandtl number Pr. The Rayleigh number Ra $=g \alpha \Delta T H^{3} / \nu \kappa$, with $g$ gravity, $\alpha$ the coefficient of thermal expansion, $H$ the height of the cavity, $\nu$ the kinematic viscosity and the diffusivity $\kappa=\nu / \operatorname{Pr}$. The results presented below were obtained with $\mathrm{Ra}=6 \times 10^{5}$ and $\operatorname{Pr}=7.5$. Distance has been non-dimensionalised using $H$ and time using $H^{2} /\left(\kappa \mathrm{Ra}^{1 / 2}\right)$, which is the boundary layer start-up time.

The temperature equation is discretised using the same approach as that used for the momentum equations, and is solved at each time step prior to the solution of the momentum/pressure correction equations. This allows the temperature in the buoyancy term to be located at the centered $n+1 / 2$ time location. As the temperature depends only on the $n$ and $n-1$ level velocities the solution of the temperature equation is uncoupled from the solution of the momentum/Poisson pressure and pressure correction equations for the $n+1$ level velocities and the pressure. The accuracy analysis developed in the previous section for the momentum/Poisson pressure and pressure correction equation will therefore also apply in this case, with the only influence of temperature being the additional second order error associated with the buoyancy term.

Initially the fluid in the square cavity is stationary and isothermal at temperature $T=0$. At time $t=0$ the left and right walls are instantaneously heated and cooled to $\Delta T / 2$ and $-\Delta T / 2$ respectively, with the top and bottom boundaries having zero normal temperature gradient. All boundaries are no-slip. The results presented below were obtained with $\operatorname{Ra}=6 \times 10^{5}$ and $\operatorname{Pr}=7.5$.

A $50 \times 50$ uniform mesh has been used. The $50 \times 50$ solution was 
compared to that obtained on a $200 \times 200$ mesh and the variation was found to be less than one percent. The $50 \times 50$ mesh is therefore considered to provide a sufficiently accurate resolution for this flow. To test the behaviour of the methods the flow was integrated from $t=0$ to $t=2$ for time-steps in the range $\Delta t=0.003125$ to 0.1 , and the 'error' expressed as the $L_{2}$ norm of the difference between a test solution obtained at a given $\Delta t$ and a benchmark solution obtained with a time step of $\Delta t=7.8125 \times 10^{-4}$, also integrated from $t=0$ to $t=2$. Total time to steady state for the cavity is orders of magnitude greater than the boundary layer start up time. The maximum time step selected, $\Delta t=0.1$, was chosen to be near to the empirically obtained stability limit of $\Delta t=0.2$.

For each of the methods and time steps results have been obtained with convergence criterion ranging from $10^{-4}$ to $10^{-11}$ in order-of-magnitude steps. The solution was considered converged at each time step when the integral over the domain of the absolute residual of the continuity equation was less than the convergence criterion. In this way it was possible to determine which was the appropriate convergence criterion for each method and time step to ensure that as accurate as possible a solution was obtained. The results presented are those for which a further reduction of the convergence criterion by an order of magnitude led to a less than one percent change in the solution accuracy. This degree of accuracy was obtained with different criteria for each method and each time step, ranging from $10^{-4}$ for the standard $\mathrm{P} 1$ method at time step $\Delta t=0.1$ to $10^{-9}$ for the iterative method at time step $\Delta t=0.003125$. For brevity only the pressure results are presented below, velocity and temperature results may be found in Armfield and Street, [13], and a complete description of the development of this flow in Patterson and Armfield [15], and Armfield and Patterson [16].

Figure 1(a) displays the pressure error plotted against time-step 

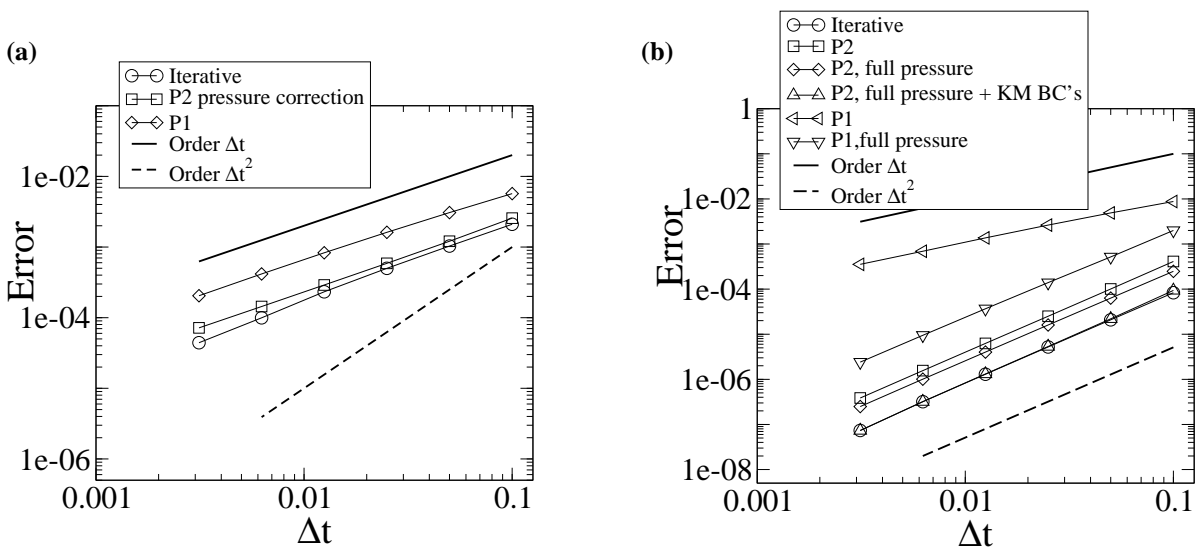

Figure 1: (a) Pressure accuracy obtained using $n+1 / 2$ test solution. (b) Pressure accuracy with pressure extrapolated to $n+1$ time location as test solution

size $\Delta t$ for each of the basic methods for the $n+1 / 2$ time location pressure. All of the schemes are showing first order in time behaviour. Shifting to the full pressure for the P1 method, and full pressure and/or Kim and Moin boundary conditions for the P2 method does not affect the first order behaviour or significantly improve the accuracy [13].

Figure 1(b) contains the results obtained when the pressure is extrapolated to the $n+1$ time location. Both the iterative and P2 methods now give second-order in time accuracy. Using the full pressure and Kim and Moin boundary conditions for the P2 method is now seen to increase the accuracy of the pressure to give approximately the same accuracy as the iterative scheme. The standard P1 method still gives only first-order accuracy as a result of the first order term on the right hand side of equation (19). When this term is included in the pressure the resultant full pressure, extrapolated to the $n+1$ location, is second-order accurate in time. 
Considering only the extrapolated $n+1$ results the full pressure P1 method is the least accurate of the second order results, while the extrapolated iterative method is the most accurate. The standard P2 and full pressure P2 results lie between the P1 and iterative results, while the full pressure P2 results with the Kim and Moin boundary conditions provide equivalent accuracy to the iterative scheme.

Figure 2 contains a comparison of the efficiency of each of the second order results. It is seen that the comparative accuracy of the methods does not necessarily predict the comparative efficiency of the schemes. The most efficient is the P2 method with Kim and Moin boundary conditions and full pressure. The least efficient overall is the P1 method. The iterative scheme is also relatively inefficient for large errors, while for small errors it is approximately the same as that of the P2 method with standard boundary conditions.

\section{Conclusions}

Iterative and non-iterative P2 fractional step solvers of the type described above produce a pressure which is second-order accurate in time. Previous investigations, which have found the pressure to be first order accurate, did so because the pressure accuracy was assumed to be located at the $n+1$ location, whereas the pressure is correctly located at the $n+1 / 2$ time level. Using an extrapolated $n+1$ pressure the second-order in time accuracy of the pressure field is evident. This does not mean that such an extrapolation is necessary to produce second-order in time accuracy, it is only required to demonstrate it at the $n+1$ location. The pressure at the $n+1 / 2$ location is therefore second-order in time.

The P1 method provides only first-order in time accuracy for 


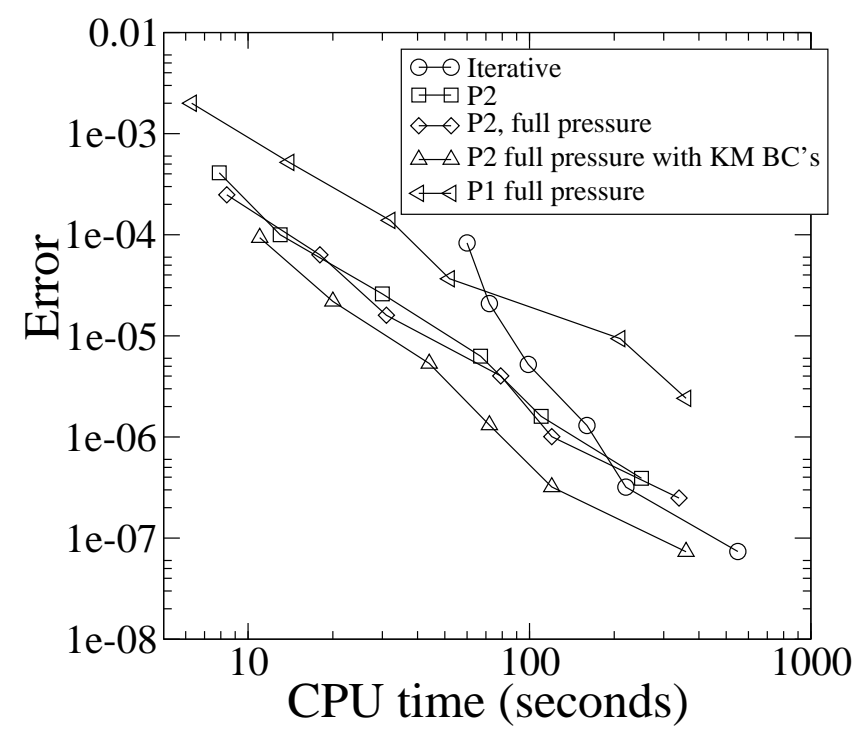

FiguRE 2: Efficiency of second order results.

the pressure field with the standard pressure approximation. For this method it is necessary to include the final term on the right hand side of equation (19) into the pressure to obtain second order accuracy.

The P2 method with Kim and Moin boundary conditions using the full pressure was found to be the most efficient of the schemes tested. This is consistent with the results presented in Armfield and Street [13], where comparative efficiencies for these schemes were examined for the velocity and temperature fields. 


\section{References}

[1] F. Harlow and E. Welch, Numerical calculation of time-dependent viscous incompressible flow of fluid with free surface. Physics of Fluids 1965; 8: 2182-2189. C22

[2] A. J. Chorin, Numerical solution of the Navier-Stokes equations. Math. Comput. 1968; 22: 745-762. C22

[3] J. Kim and P. Moin, Application of a fractional step method to incompressible Navier-Stokes equations. J. Comp. Physics 1985; 59: 308-323. C22, C31

[4] Y. Zang, R. Street and J. Koseff, A non-staggered grid, fractional step method for time-dependent incompressible Navier-Stokes equations in curvilinear coordinates. J. Comp. Physics 1994; 114: 18-33. C22

[5] G. Karniadakis, M. Israeli and S. Orszag, High-order splitting methods for the Navier-Stokes equations. J. Comp. Physics 1991; 97: 414-443. C22

[6] J. B. Perot, An analysis of the fractional step method.

J. Comp. Physics 1993; 108: 249-253. C22

[7] J. Van Kan, A second order accurate pressure correction scheme for viscous incompressible flow. SIAM J. Sci. Stat. Comput. 1986; 7: 870-891. C22

[8] J. B. Bell, P. Colella, A second-order projection method for the incompressible Navier-Stokes equations. J. Comp. Physics 1989; 85: 257-283. C22

[9] P. Gresho, On the theory of semi-implicit projection methods for viscous incompressible flow and its implementation via a 
finite element method that also introduces a nearly consistent mass matrix. Part 1: theory. Int. J. Numerical Methods in Fluids 1990; 11: 587-620. C22

[10] J.-L. Guermond and L. Quartapelle, Calculation of incompressible viscous flows by an unconditionally stable projection FEM, J. Comp. Physics 1997; 132: 12-33. C22

[11] E. Y. Tau. A second order projection method for the incompressible Navier-Stokes equations in arbitrary domains. J. Comp. Physics 1994; 115: 147-152. C22

[12] J. Dukowicz and A. Dvinsky, Approximate factorisation as a high order splitting for the incompressible flow equations. J. Comp. Physics 1992; 102: 336-347. C22

[13] S. Armfield and R. Street, An analysis and comparison of the time accuracy of fractional step methods for the Navier-Stokes equations on staggered grids, Int. J. for Numerical Methods in Fluids 2002; 38: 255-282. C22, C31, C34, C35, C37

[14] B. P. Leonard, A stable and accurate convective modelling procedure based on quadratic upstream interpolation. Comp. Meth. Appl. Mech. and Eng. 1979; 19: 59-98. C31

[15] J. C. Patterson and S. W. Armfield, Transient features of natural convection in a cavity. J. Fluid Mech. 1990; 219: 469-497. C34

[16] S. W. Armfield and J. C. Patterson, Wave properties of natural convection boundary layers. J. Fluid Mech. 1992; 239: 195-211. C34 